\title{
Preliminary evidence for an influence of exposure to polycyclic aromatic hydrocarbons on the composition of the gut microbiota and neurodevelopment in three-year-old healthy children
}

\author{
Wei Zhang ${ }^{1 \dagger}$, Zhongqing Sun ${ }^{2 \dagger}$, Qian Zhang ${ }^{3 \dagger}$, Zhitao Sun ${ }^{4}$, Ya Su', Jiahui Song ${ }^{1}$, Bingling Wang ${ }^{4^{*}}$ and Ruqin Gao ${ }^{4^{*}}$ (I)
}

\begin{abstract}
Background: During the second and third year after birth the gut microbiota (GM) is subjected to important development. The polycyclic aromatic hydrocarbon (PAH) exposure could influence the GM in animal and early postnatal exposure is associated with neurodevelopment disorder in children. This study was designed to explore the possible influence of the polycyclic aromatic hydrocarbons (PAHs) on the composition of the gut microbiota (GM) and neurodevelopment in a sample of 38 healthy children at the age of 3 years.

Methods: A brief development (Gesell Development Inventory, GDI) and behavior test (Child Behavior Checklist, $\mathrm{CBCL}$ ) were completed on 3-yr-olds and stool samples were collected for $16 \mathrm{~S}$ rRNA V4-V5 sequencing. The PAHDNA adduct in the umbilical cord blood and the urinary hydroxyl PAHs (OH-PAHs) at the age of 12 months were measured as pre- and postnatal PAH exposure, respectively.

Results: The most abundant two phyla were Bacteroidetes (68.6\%) and Firmicutes (24.2\%). The phyla Firmicutes, Actinobacteria, Proteobacteria, Tenericutes, and Lentisphaerae were positively correlated with most domain behaviors of the GDI, whereas the Bacteroidetes, Cyanobacteria, and Fusobacteria were negatively correlated. Correspondingly, the phyla Bacteroidetes, Actinobacteria, and Fusobacteria showed positive correlations with most CBCL core and broadband syndromes, whereas the Firmicutes, Verrucomicrobia, Synergistetes, Proteobacteria and Tenericules were negatively correlated. The OH-PAH levels were not significantly associated with the Firmicutes phylum whereas the Bacteroidetes, Bacteroidia, and Bacteroidales all showed significant negative association with the OH-PAH levels.

Conclusion: The current findings suggest that composition of the GM is associated with neurodevelopment of the child. PAHs seem to change the relative abundance of some taxa (some deleted and some recruited) to counteract the negative effects of the PAHs.
\end{abstract}

Keywords: Children, Gut microbiota, Polycyclic aromatic hydrocarbons, Neurodevelopment

\footnotetext{
*Correspondence: binglingw@yeah.net; gaoruqin@yeah.net

'Wei Zhang, Zhongqing Sun and Qian Zhang contributed equally to this work.

${ }^{4}$ Department of Environmental Health, Qingdao Municipality Center for Disease Control and Prevention, Qingdao Institute of Preventive Medicine, Qingdao 266033, China

Full list of author information is available at the end of the article
}

C C The Author(s). 2021 Open Access This article is licensed under a Creative Commons Attribution 4.0 International License, which permits use, sharing, adaptation, distribution and reproduction in any medium or format, as long as you give appropriate credit to the original author(s) and the source, provide a link to the Creative Commons licence, and indicate if changes were made. The images or other third party material in this article are included in the article's Creative Commons licence, unless indicated otherwise in a credit line to the material. If material is not included in the article's Creative Commons licence and your intended use is not permitted by statutory regulation or exceeds the permitted use, you will need to obtain permission directly from the copyright holder. To view a copy of this licence, visit http://creativecommons.org/licenses/by/4.0/ The Creative Commons Public Domain Dedication waiver (http://creativecommons.org/publicdomain/zero/1.0/) applies to the data made available in this article, unless otherwise stated in a credit line to the data. 


\section{Background}

The period of rapid brain growth spans from the 3rd trimester of pregnancy to at least 2 years after birth [1]. This rapid-growth period is a window of opportunity in which foundational processes such as proliferation and migration of glia, myelination of axons, and synaptogenesis are occurring and extremely plastic and can be easily subjected to remodeling in response to some environmental inputs, with subsequent influence on behavior and learning processes [2]. Simultaneously, the first 3 years of life seems to be critical for the assembly of the commensal ecosystem; during the second and third year the gut microbiota (GM) is subjected to important development, becoming more similar to the adult ecosystem [3].

Recent research suggests that the GM, a complex microbial ecosystem populating our gastro-intestinal tract, may be a key modulator of neurodevelopment through the microbiome-gut-brain axis $[4,5]$. In rodents, experimental altering of the GM impacts anxiety- and depression-related behaviors in multiple well-established paradigms [6-9]. Cognitive effects have also been reported [10-12]. In humans, altered composition of the GM has been reported in children with psychiatric diseases such as autism [13-16] and attention-deficit/ hyperactivity disorder [17], and has been linked to childhood temperament [18]. Besides postnatal environmental factors, prenatal influences like maternal factors [19], gestational exposures [20-23], and mode of delivery [24, 25], were all associated with the diversity and architecture of the infant's GM during the first year of life.

Prenatal polycyclic aromatic hydrocarbon (PAH) exposure produced neurodevelopmental toxicity in both animal experiments [26-28] and human epidemiological studies [29-32]. Our previous functional studies showed that organic extracts from settled house dust could interfere with human thyroid-hormone metabolism by competitively antagonizing thyroid-hormone receptors [33]. More importantly, our previous study found that PAHs, especially high-molecular-weight PAHs in settled house dust were significantly correlated with children's intellectual development and neurobehavioral problems. Additionally, PAH concentrations were positively correlated with severity of children's neurobehavioral problems characterized by compulsivity and aggressivity [34]. These findings suggested a possible relationship between early indoor exposure to PAHs and children's later neurobehavioral development, and consequently, intellectual and neurodevelopmental disorders. Our prior investigation showed that exposure to PAHs, represented by 10 hydroxyl metabolites, in toddlers at 12 months could influence their neurodevelopment.

Persistent organic pollutants (POPs) can change the diversity of the GM by activating aromatic hydrocarbon receptors (AhR), affect the host's metabolic balance [35, 36], and further participate in the regulation of neurodevelopmental disorders-related behavior and physiological abnormalities [7]. As one of the POPs, PAHs are important ligands for AhR in the body. The load of PAHs in early childhood mainly comes from the digestive tract, and most of the PAHs, especially high molecular weight PAHs, are mainly excreted from the digestive tract through feces, not through the kidney [37]. Interestingly, our team's findings show that PAHs in urban house dust that cause internalizing behavior problems are more likely to be of high molecular weight [34]. Therefore, we suspected a possible important role of the GM in the neurodevelopmental toxicity of PAHs.

Some psychiatric diseases like autism are extremely heterogeneous neurodevelopmental disorders and their onset can vary, with some children showing signs from early infancy and others exhibiting symptoms of regression at the age of 2-3 years. Notably, the first 3 years of life seems to be critical for the assembly of the commensal ecosystem. The fascinating observation of a critical period in which the microbiota could affect neurodevelopment and the existence of sensitive periods for pollutant-dependent refinement of sensory modalities (i.e., vision, hearing etc.), language acquisition, fear extinction and many others processes, suggests the appealing possibility of microbiome-driven stimuli involvement in fine-tuning neuronal circuits remodeling during early age. The present study is designed to explore the possible association between the composition of the GM and neurodevelopment in a sample of healthy 3-yr-old children. Simultaneously, early postnatal PAH exposure was measured to explore the possible influence on the GM.

\section{Methods \\ Participants}

The infants were selected from our prospective birth cohort established in 2014 [38, 39]. Pregnant women at the obstetrics clinic of the hospitals in Qingdao were invited to sign the informed-consent form for participation in this prospective cohort study. In order to avoid the possible influence of the mode of delivery, the type of feeding (breast or formula milk) and the use of antibiotics on the composition of the neonatal gut microbiota, additionally to meeting the criteria of eligibility of the cohort, the subjects included in the present study should be natural delivery, breastfeeding and have been free of antibiotic use in the preceding 30 days. In the end, only 38 had postnatal PAH exposure levels and underwent GM measurement. This study was approved by the Ethics Committee and Institutional Review Board of Qingdao Municipal Center for Disease Control and Prevention. The authors declare that all procedures 
contributing to this work comply with the ethical standards of the relevant national and institutional committees on human experimentation and with the Helsinki Declaration of 1975, as revised in 2008.

\section{S rRNA gene sequencing}

Fresh fecal samples were collected from recruited subjects at 3 years of age and transported to Qingdao CDC laboratory with an ice packs within $2 \mathrm{~h}$. All samples were then immediately frozen and stored at $-80^{\circ} \mathrm{C}$ prior to analyses. The diversity and abundance of the GM were analyzed by $16 \mathrm{~S}$ rRNA gene sequencing (V4-V5 region) with the Illumina MiSeq. DNA of the GM from stool was obtained by the QIAamp DNA Stool Mini Kit (Qiagen, Germantown, MD). Then, the DNA samples were sent to the TinyGene Company (Shanghai, China) for $16 \mathrm{~S}$ rRNA gene-sequencing analysis.

\section{Postnatal PAH-exposure measurement}

Morning first-void urine samples were collected for 5 consecutive days when the infants were able to urinate spontaneously (between 1.5 and 2 years old). The pooled sample of 5 consecutive days was used for the postnatal PAH measurement. Urinary hydroxyl $\mathrm{PAH}$ testing was performed following the method described by Guo et al. with minor changes [40] (Supplementary information). Our selected hydroxyl metabolites of PAHs included 10 exposure biomarkers which were: 1-, 2-hydroxynaphthalene; 2hydroxyfluorene; 1-, 2-, 3-, 4-, 9-hydroxyphenanthrene; 1hydroxypyrene; and 6-hydroxychrysene (Supplementary information).

\section{Measures of child neurodevelopment and behavior}

The mothers of 2-3-yr-olds were asked to fill the Child Behavior Checklist (CBCL) to evaluate the children's behavior [41]. The Chinese version of CBCL/2-3 has been translated and applied in China with a Cronbach $\alpha=$ 0.78 , and validity coefficient $=0.73$ [42]. The checklist contains six core syndromes: Anxious/depressed; Withdrawn; Sleep problems; Somatic complaints; Aggressive behavior; and Destructive behavior. In addition, there are two broadband syndromes: Internalizing and Externalizing.

The Gesell Development Inventory (GDI) was selected in the present study for the measures of child neurodevelopment. The GDI has been translated and standardized by the Beijing Children's Health Care Institute, and consists of five behavioral domains: Adaptive; Gross motor; Fine motor; Language; and Personal social behaviors [43]. Each child was assigned a developmental quotient (DQ) in each of the five specific domains. The higher the $\mathrm{DQ}$, the better the neurodevelopment. All subjects were tested by a professional doctor at the
Department of Child Health Care at Huangdao Maternity and Child Care Hospital of Qingdao.

\section{Statistical analyses}

All microbiome data were represented as proportions of total microbiome. Mean and standard deviation (SD) were used to describe the distribution of DQs and median and range were used for CBCL scores. Half of the limit of detection (LOD) was used for the OH-PAHs for the final statistical analyses. Due to small sample size and non-normal distributions, Spearman correlations examined the association between specific GM proportions and DQs and CBCL Scores. R-statistics (http://www.rproject.org/) was used for hierarchical clustering analysis and heatmaps of the Spearman correlation coefficient data to give insight into the structure of the data. Higher DQ and lower CBCL scores reflect better neurodevelopment. OH-PAHs were divided into high exposure (> $30 \mu \mathrm{g} / \mathrm{g}$ creatinine $)$ and low exposure $(\leq 30 \mu \mathrm{g} / \mathrm{g}$ creatinine) to explore the PAH influence on the GM. We further performed partial correlation analysis for the association between DQ and GM proportions with controls for $\mathrm{OH}-\mathrm{PAH}$ and sex. All statistical analyses were performed using IBM SPSS 21.

\section{Results}

Characteristics of the study population

Of 38 children, 20 were boys (52.6\%). Their average gestational age was $39 \pm 1$ weeks, and the average birth weight was $3604 \pm 733 \mathrm{~g}$. The mean DQs for all five behavior domains were all above 100 . The medians for six CBCL core syndromes and two broadband syndromes were all less than 10 . The geometric mean (95\% confidence interval) for OH-PAH ( $\mu \mathrm{g} / \mathrm{g}$ creatinine) was 10.23 $(6.44,16.25)$ (Table 1).

\section{Association between GM and neurodevelopment}

The most abundant GM phyla were Bacteroidetes and Firmicutes with mean \pm SD relative abundance $0.6862 \pm$ 0.0940 and $0.2420 \pm 0.0805$, respectively (Figure S1). The phyla of Firmicutes, Actinobacteria, Proteobacteria, Tenericutes, and Lentisphaerae were positively correlated with most domain behavior scores of the GDI. Firmicutes showed a significant correlation with Gross motor behavior score $(r=0.327, P<0.05)$. Bacteroidetes, Cyanobacteria, and Fusobacteria showed negative correlations with most domain behaviors, of which Bacteroidetes showed a significant correlation with the Gross motor behavior score $(r=-0.416, P<0.01)$ and Fusobacteria with the Adaptive behavior score $(r=-0.334, P<0.05)$ (Fig. 1).

Consistent with the phylum level, the lower taxa Bacteroidia, Bacteroidales, Myroides, Bacteroides stercoris, Prevotella buccae, Myroides sp. A21 and Myroides 
Table 1 The general information, DQs and CBCL scores of the subjects $(n=38)$

\begin{tabular}{|c|c|}
\hline Variables & Subjects \\
\hline \multicolumn{2}{|l|}{ Mother's age(y) } \\
\hline Mean \pm SD & $34 \pm 3$ \\
\hline Range & $27 \sim 39$ \\
\hline \multicolumn{2}{|l|}{ Gestational weeks(w) } \\
\hline Mean \pm SD & $39.62 \pm 1.01$ \\
\hline \multicolumn{2}{|l|}{ Child's birth weight(g), mean \pm SD } \\
\hline Mean \pm SD & $3604.17 \pm 733.33$ \\
\hline \multicolumn{2}{|l|}{ DQs, Mean \pm SD } \\
\hline Adaptive & $102 \pm 6$ \\
\hline Gross motor & $108 \pm 7$ \\
\hline Fine motor & $108 \pm 8$ \\
\hline Language & $103 \pm 7$ \\
\hline Personal social & $106 \pm 7$ \\
\hline CBCL syndromes, median (range) & $(n=25)$ \\
\hline Anxious/depressed & $3(0,13)$ \\
\hline Withdrawn & $3(0,14)$ \\
\hline Sleep problems & $2(0,8)$ \\
\hline Somatic complaints & $3(0,16)$ \\
\hline Aggressive & $4(0,19)$ \\
\hline Destructive & $3(0,11)$ \\
\hline Internalizing & $5(0,23)$ \\
\hline Externalizing & $7(0,26)$ \\
\hline Total CBCL score & $24(4,95)$ \\
\hline \multicolumn{2}{|l|}{$\mathrm{OH}-\mathrm{PAH} / \mathrm{Cr}(\mu \mathrm{g} / \mathrm{g} \mathrm{Cr})$} \\
\hline GM $(95 \% \mathrm{Cl})$ & $10.23(6.44,16.25)$ \\
\hline
\end{tabular}

$S D$ standard deviation, $\mathrm{Cr}$ creatinine, $G M$ geometric mean, $\mathrm{Cl}$ confidence interval

odoratus of the Bacteroidetes showed significant negative correlations with some domain behaviors of the GDI (Figures S2-S6). For the Firmicutes phylum, the Ruminococcaceae, Christensenellaceae, Ruminococcus, Ruminiclostridium, Anaerotruncus, Hungatella, Ruminococcus sp N15MGS57, Bacterium enrichment culture clone Ecwsrb027, Clostridium lavalense, Uncultured prokaryote, Ruminococcaceae bacterium, Uncultured Veillonellaceae bacterium, Bacterium enrichment culture clone Ecwsrb027, Uncultured Veillonellaceae bacterium, Eubacterium ramulus, Bacterium YE57, Christensenella minuta and Streptococcus mutans showed significant positive correlations with some domains of the GDI (Figures S2-S6).

Contrary to the positive direction of the DQs, the lower the CBCL score the better the neurodevelopment. For the phyla Bacteroidetes, Actinobacteria, and Fusobacteria, positive significant correlations were between Bacteroidetes and Withdrawn syndromes $(r=0.551, P<$
$0.01)$, and Somatic complaints $(r=0.413, P<0.05)$. For Actinobacteria, positive correlations were found with Destructive behavior $(r=0.589, P<0.01)$ and Externalizing behavior $(r=0.471, P<0.05)$. The phyla Firmicutes, Verrucomicrobia, Synergistetes, Proteobacteria and Tenericules showed significant negative correlations on CBCL between Proteobacteria and Withdrawn syndromes $(r=-0.435, P<0.05)$, Verrucomicrobia and Anxious/depressed $(r=-0.476, P<0.05)$, Aggressive $(r=-$ $0.542, P<0.05)$, Destructive $(r=-0.552, P<0.01)$, Internalizing $(r=-0.471, P<0.05)$, Externalizing behaviors $(r=-0.598, P<0.01)$, and the total CBCL scores $(r=-$ 0.410, $P<0.05)$ (Fig. 2).

Consistent with the phylum level, the Bacteroidia, Bacteroidales, Bacteroides, Bacteroides vulgatus, Bacteroides intestinalis, Bacteroides uniformis, and Bacteroides caccae within Bacteroidetes phylum all showed significant positive correlation with most CBCL behavior syndromes (Figures S7-S11). Within Firmicutes, the Anaerostipes, Oscillospira, Lactobacillus, Caproiciproducens, Selenomonas, Ezakiella, Moryella, Acetitomaculum, gut metagenom, and uncultured rumen bacterium all showed negative correlations with most CBCL behavior syndromes. As with most lower taxa of the Firmicutes phylum, within Verrucomicrobia, all detected lower taxa showed negative correlations with all CBCL behavior syndromes.

Despite showing mostly consistency, the lower taxa within the phylum still showed some variance. For example, within the phylum Bacteroidetes, the family Bacteroidaceae showed the same associations as the order Bacteroidales, but the family Prevotellaceae showed negative correlations with almost all CBCL behavior syndromes (Figure S9).

\section{Influence of the PAH exposure on GM and neurodevelopment}

We explored the influence of the PAH exposure on the relative abundance of the GMs. Within the Bacteroidetes phylum, the microbiota showed negative association with the OH-PAH levels, of which the Bacteroidia class, the Bacteroidales order, and the Barnesiella genus showed statistical significance (Fig. 3). The Firmicutes phylum seemed to show positive association although no statistical significance was found. After we divided the $\mathrm{OH}-\mathrm{PAH}$ levels into high- and low-exposure groups, of the lower taxa, the Negativicutes class, the Selenomonadales and Bacillales order, the Lactobacillaceae family and the Lactobacillus genus all showed significantly higher relative abundance in high-exposure group, whereas the Clostridium perfringens, the Clostridium Colinum, and the Clostridium sp 73 54FAA species all showed lower relative abundance (Fig. 4). Additionally, Roseburia, Ruminococcus, Blautia, Dialister, 


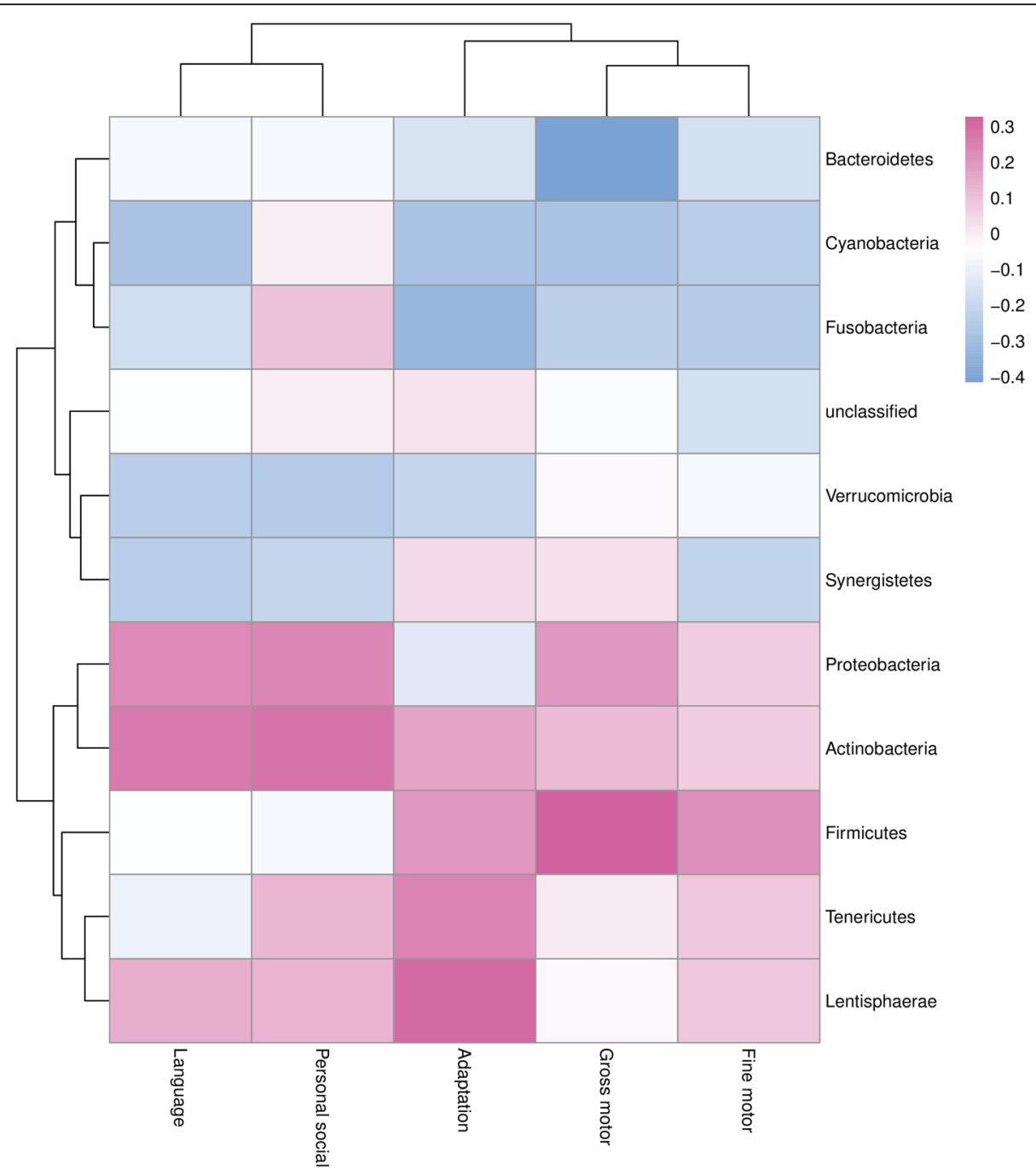

Fig. 1 Heatmap of the Spearman correlation coefficients between scores of five GDI behavior domains and abundance of 11 GM phyla. Colorcoded with blue for negative lower coefficients and red for positive higher coefficients. Dendrograms present clustering of GM phyla (rows) and GDI behavior domains (columns), which is based on hierarchical clustering with Euclidean distance metric and average linkage

Coprococcus, Megasphaera, Eubacterium, and Anaerostipes all showed negative association with the postnatal PAHs exposure levels (Figures S10-S11).

Although the Bacteroidetes phylum, the Bacteroidia class, and the Bacteroidales order all showed negative association with $\mathrm{OH}-\mathrm{PAH}$ levels and all GDI domain behaviors (Fig. 3), and positive association with all CBCL behavior syndromes (Fig. 4), the Bacteroides nordii, Prevotella buccae and Eubacterium siraeum species all showed positive association with $\mathrm{OH}-\mathrm{PAH}$ levels, but indicated negative correlation with most CBCL behavior syndromes (Fig. 4). It is interesting that the unidentified bacteria at the genus level showed significant positive correlation $(r=0.453, P<0.001)$ with $\mathrm{OH}-\mathrm{PAH}$ levels and significant negative correlation with most CBCL behavior syndromes (Fig. 5).
When we further adjusted for OH-PAHs to explore the PAH exposure influence on the association between the GM and GDI, the significant correlation between Bacteroidetes and gross motor behavior disappeared (Fig. 6). After adjusting for OH-PAHs, the phylum Cyanobacteria showed a significant negative association with Adaptation $(r=-0.532, P<0.01)$, Gross motor $(r=-0.410, P<0.05)$, and Language $(r=-0.749, P<$ 0.01) domain behavior; the phylum Tenericutes showed a significant positive association with Personal social domain behavior $(r=0.395, P<0.05)$. These associations did not change much after adjustment for sex.

\section{Discussion}

The composition of the GM can have a profound impact on human health. Accumulating evidence has suggested 


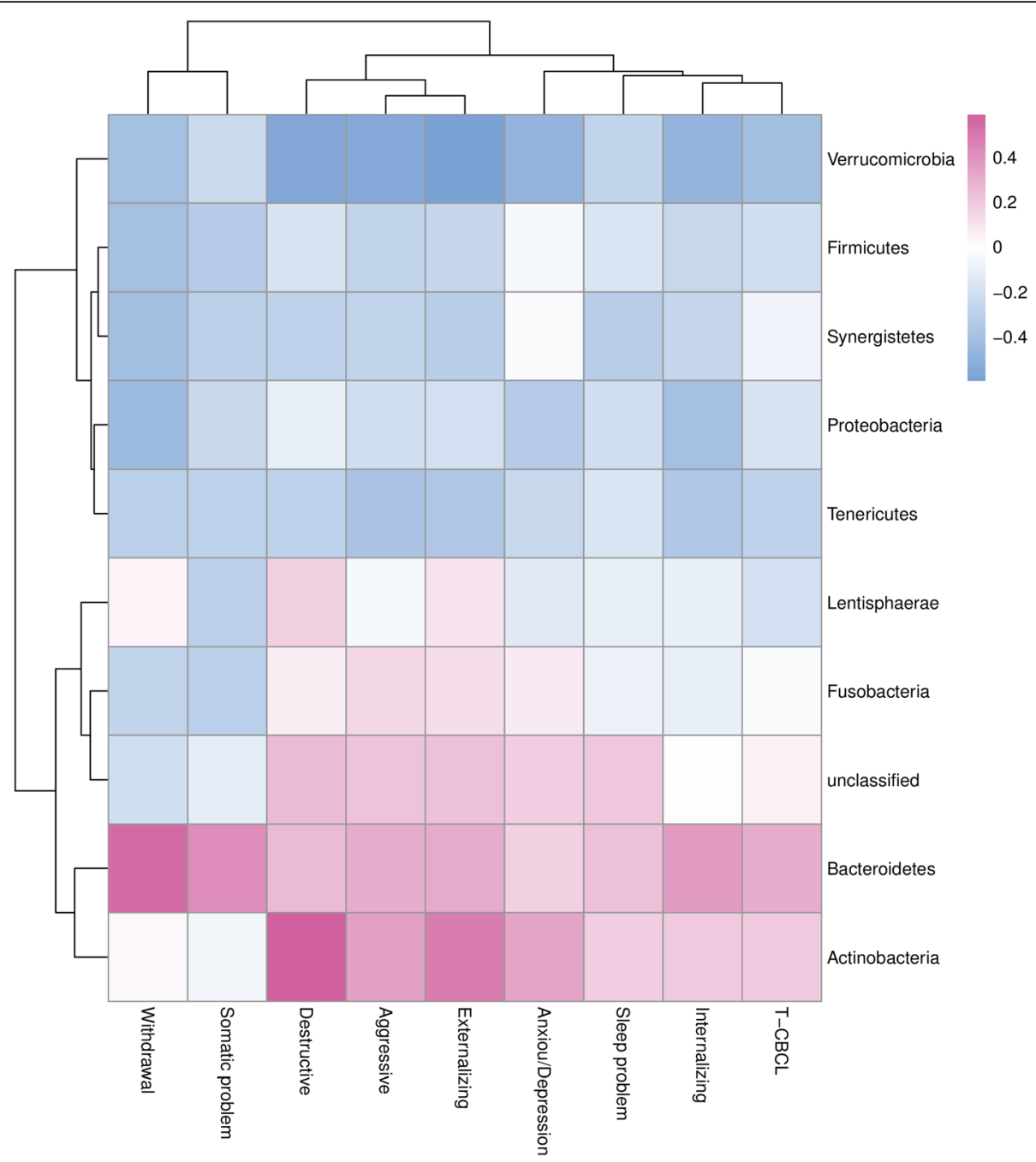

Fig. 2 Heatmap of the Spearman correlation coefficients between scores of six core syndromes, two broadband syndromes, and total Score of $\mathrm{CBCL}$ and abundance of $11 \mathrm{GM}$ phyla. Color-coded with blue for negative lower coefficients and red for positive higher coefficients.

Dendrograms present clustering of GM phyla (rows) and CBCL syndromes (columns), which is based on hierarchical clustering with Euclidean distance metric and average linkage

that environmental pollution may be associated with several pathologies [44]. In the present study, the GM composition in 3-year-old children was measured and the internal PAH concentration was analyzed to explore the possible influence on the GM and related neurobehavioral development.

A recent study investigated whether the composition of the GM at 1 year of age was associated with cognitive outcomes [45]. The cognitive outcomes were measured by the Mullen Scales of Early Learning in 89 infants at 1 year of age. The global and regional brain volumes were obtained with structural magnetic resonance imaging at 1 and 2 years. The neuroimaging data suggested that GM might have minimal effects on regional brain volumes at 1 and 2 years of age. But Mullen scores at 2 years of age differed significantly between clusters of
GM. That was the first study to demonstrate associations between the GM and cognition in human infants. However, GM was assessed at 1 year of age when intake of solid foods varies significantly, potentially resulting in rapid shifts of GM. By contrast, in the present study the GM was assessed at 3 years of age, and Bacteroidetes and Firmicutes were the top two dominant phyla. This is consistent with those reports of children in other studies [46], and in adults [47].

Multiple connections exist between the gastrointestinal tract and the brain, including immune, metabolic, circulatory, and neuronal pathways [4, 48]. Commensal GM is known to regulate various aspects of each, potentially leading to changes in host behavior. The GM can alter levels of pro-inflammatory cytokines produced by macrophages and $\mathrm{T}$ cells in the gastrointestinal tract 


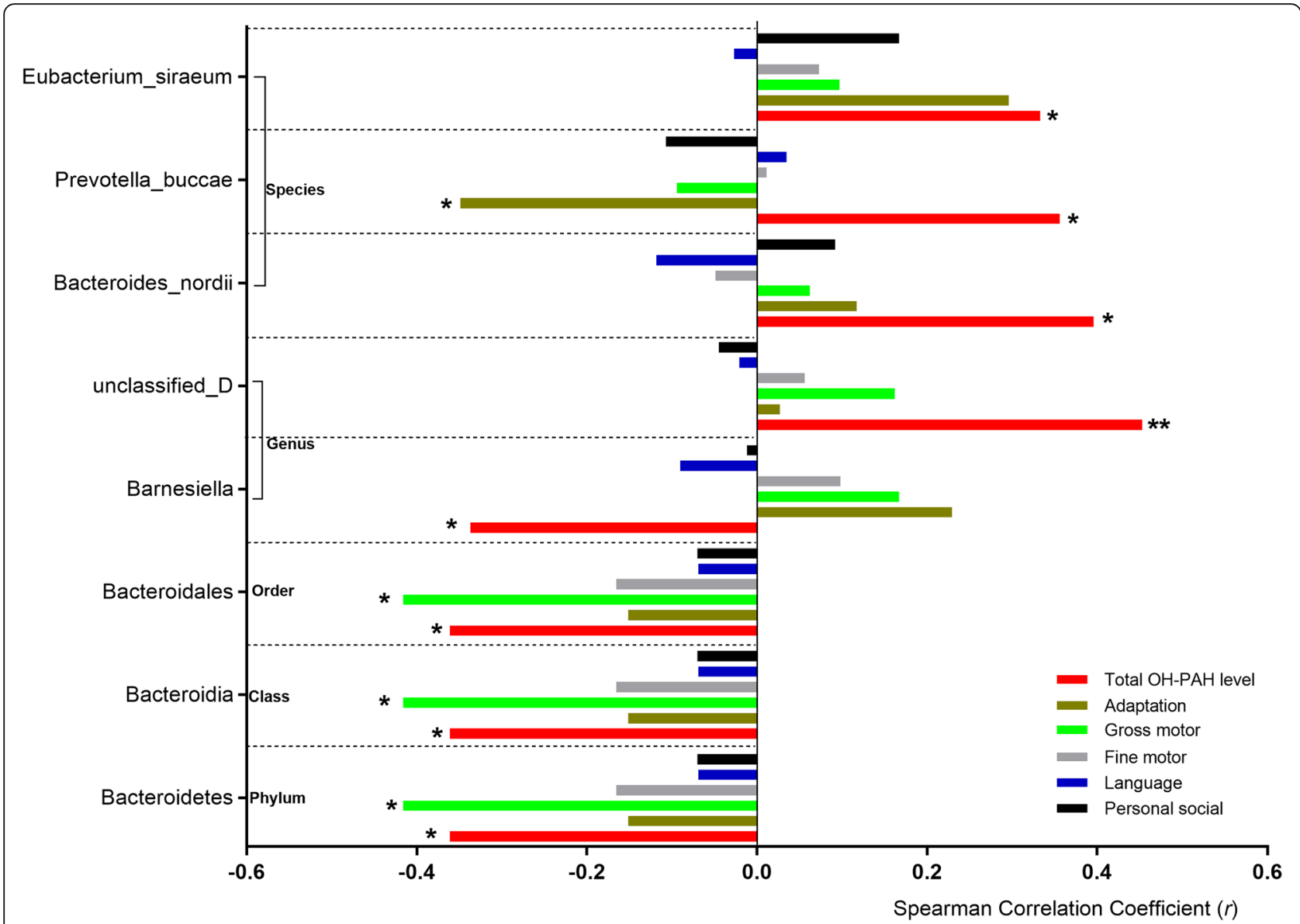

Fig. 3 The Spearman correlation coefficients between the abundance of the GM and OH-PAHs (red) and scores of five GDI behavior domains. ${ }^{*} P<0.05 ;{ }^{*} P<0.01$

[49], and microglia in the brain [50]. Microglia have been linked to behavioral changes associated with neurodegenerative disorders and may thereby be an indirect mechanism through which bacteria influence host behavior [51]. We used two behavior scales, GDI and $\mathrm{CBCL}$, to evaluate infant neurodevelopment. Higher DQs of the GDI means better development. Conversely, higher scores of the CBCLs means poorer development. Positive and negative results of the two scales indicate a better understanding of the association between the GM and the neurodevelopment.

From both GDI and CBCL scales, our results indicated that higher proportions of Firmicutes and Verrucomicrobia at the phylum level may in some way be beneficial or protective for the development of neural system, whereas higher proportions of Bacteroidetes may be less beneficial, or possibly deleterious. Consistent with our results are reports of negative associations between proportion of Bacteroidetes and cognitive impairment in persons with severe liver dysfunction [52] and positive associations between Verrucomicrobium and learning and memory performance in the microbiome depletion/ transplantation mouse model [53].

At the lower taxa of the phylum level, the GM composition shifts seem sophisticated. Some orders of the Bacteroidetes and Firmicutes showed consistent association; this was also found in a mouse model with dietcaused changes in the GM and cognitive flexibility [54]. In that study, both increased Clostridiales order of the Firmicutes phylum and lower percentages of Bacteroidales were found to be related to searching more for the old platform position than the normal animals.

No consensus has emerged from previous human studies of mental disorders and GM concerning which bacterial taxa are most relevant. However, species from the Lactobacillus have been used as a therapeutic intervention to suppress depressive-like behaviors in animal models $[55,56]$. In the present study, the Lactobacillus genus, and Lactobacillus delbrueckii species were all negatively correlated with CBCL behaviors. The genera of the Eggerthella, Halomonas, and Turicibacter indicated positive association with the CBCL behaviors, 


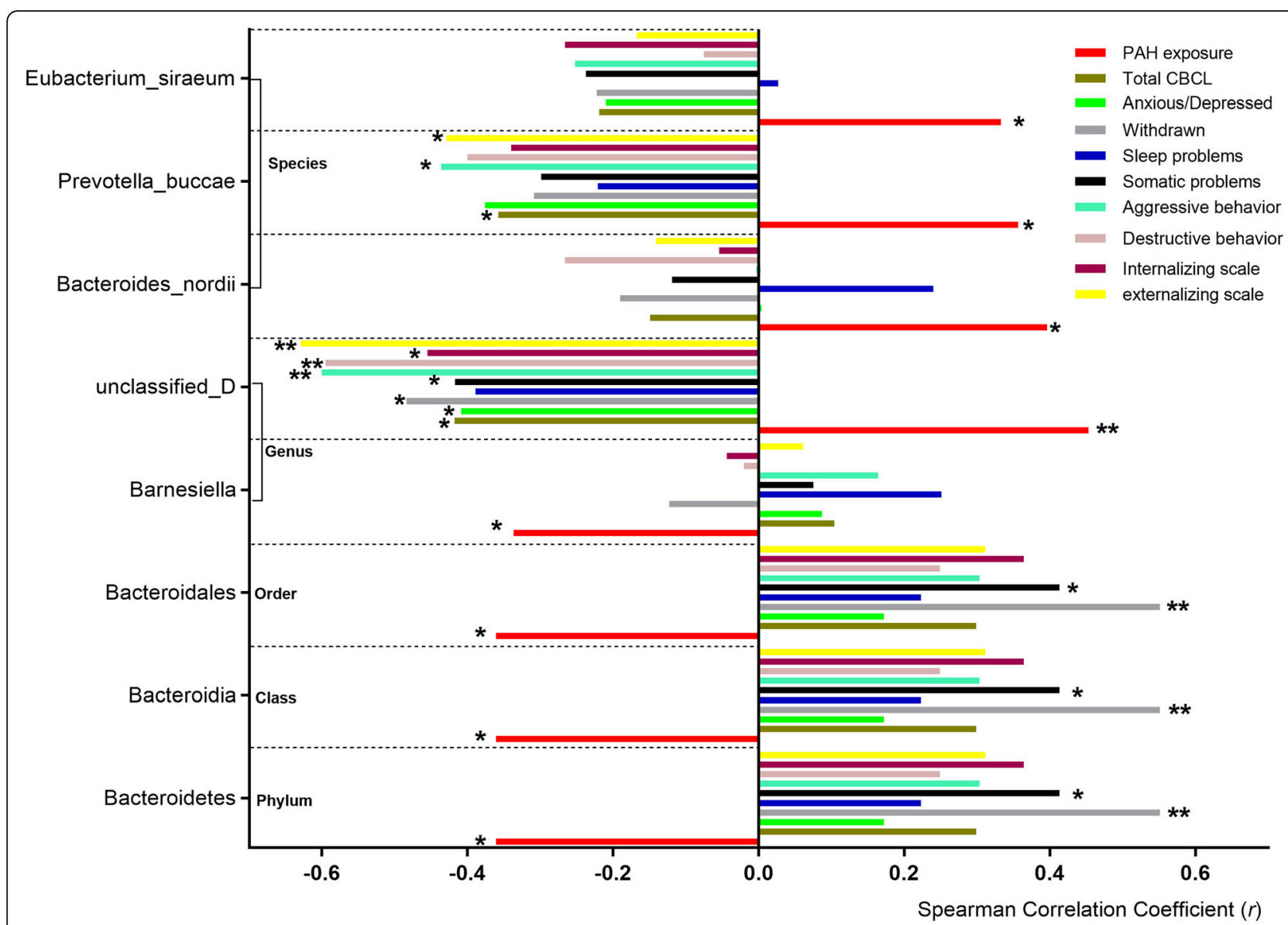

Fig. 4 The Spearman correlation coefficients between the abundance of the GM and OH-PAHs (red) and scores of the CBCL behavior syndromes. ${ }^{*} P<0.05$; ${ }^{*} P<0.01$

which is consistent with the changes in a rat model of major depression [57]. Our results showed that Bacteroides coprocola species correlated negatively and Bacteroides uniformis correlated positively with the CBCL behavior syndromes; this is consistent with results reported in Attention-deficit/hyperactivity disorder (ADHD) [58]. The beneficial effects of the Akkermansia and Lactobacillus genera found in the present study were consistent with those reported in a recently published meta-analysis for association between the GM and Autism Spectrum Disorder [59].

PAHs are considered to be high-priority environmental contaminants not only because of their toxic, carcinogenic, and putative estrogenic or antiestrogenic properties in humans $[60,61]$ but also because of neurodevelopmental toxicity in children [29-32]. Ingested PAHs reach the intestine enterocytes and liver hepatocytes and act as AhR ligands. Moreover, microbiota in the colon can catalyze the conversion of PAHs to estrogen. This bioactivation could be an underlying mechanism of PAH toxicity [61].
Oral exposure to Benzo(a) pyrene (BaP)-inducing gut microbial shifts in a murine model has been reported [62]. Contrary to our results, that study reported that after 28 days of subchronic oral exposure to $\mathrm{BaP}$, there was an increase in the pro-inflammatory bacterial taxa belonging to the families Alcaligenaceae, Bacteroideceae, Erysipelotrichaceae, Paraprevotellaceae, Porphyromonadaceae and Turicibacter. In contrast, populations of the anti-inflammatory bacterial taxa including Lactobacillaceae, Lachnospiraceae, Ruminococcaceae, and Verrucomicrobiaceae were reduced [62]. Our results seemed more concordant with the transient protective effects against the pro-inflammatory proprieties of $\mathrm{BaP}$ at the beginning of the experiment. After the first $\mathrm{BaP}$ oral administration, an increase in relative abundance of beneficial GM Akkermansia muciniphila was observed. This apparent trend was also found in our results although with no statistically significant difference.

Although we did not confirm our hypothesis at the high taxa, we did find negative associations of PAH levels with the Roseburia, Ruminococcus, Blautia, 


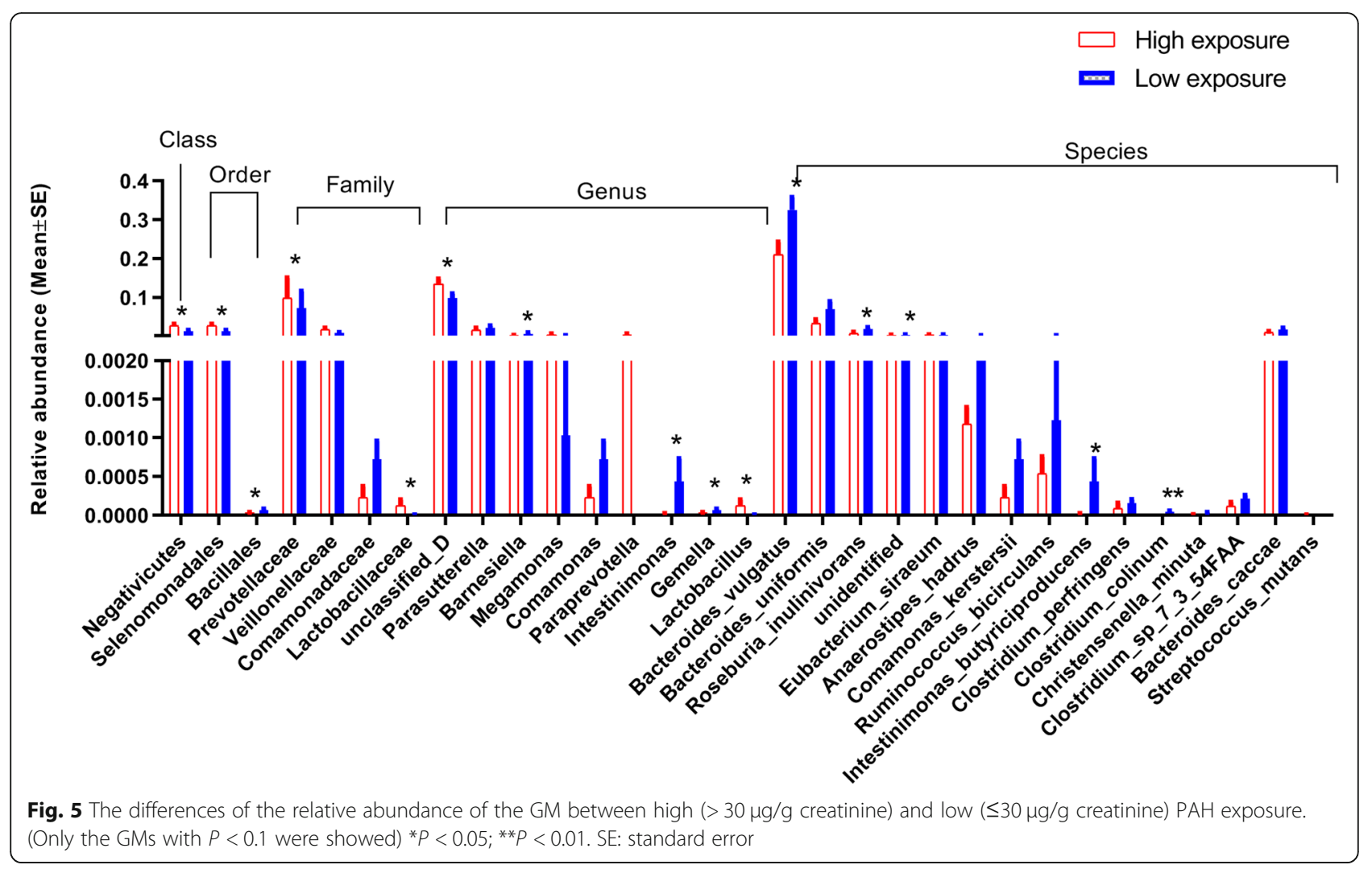

Dialister, Coprococcus, Megasphaera, Eubacerium and Anaerostipes, which were related to the gut production of the microbial metabolites propionate, acetate, or butyrate. As the members of the short chain fatty acids, propionate, acetate, and butyrate were depleted in the patients with major depressive disorder (MDD) through potential mechanisms of epigenetics (HDACi and DNA methylation modulator) or receptors like GPR43 and GPR41 [63].

Our results seemed more consistent with another two AhR modulators, 2, 3, 7, 8-tetrachlorodibenzo-p-dioxin (TCDD) [64], and polychlorinated biphenyls (PCBs) [65], which have been found potentially to shape the gut microbiome composition in mice. TCDD or PCB administration dramatically increased the abundance of Firmicutes and decreased the abundance of Bacteroidetes in mice. At the family level, the levels of Lactobacillaceae and Desulfovibrionaceae significantly increased and the levels of Prevotellaceae decreased in the feces after TCDD exposure.

Our results also indicated that the Lactobacillus genus of the Lactobacillaceae family positively associated with PAH levels. The Lactobacillus genus has been shown to produce acetylcholine (Ach), another kind of microbial metabolite $[4,63,66]$. Ach is essential for memory-trace formation and long-term memory, and Ach level reflects acetylcholine esterase (AchE) activity. AchE regulates signal transmission among cholinergic brain neurons by degrading Ach. AchE activity has been reported to decrease significantly in regions with high air concentrations of PAHs [67]. Serum Ach increase was also reported in a population of Chinese coke-oven workers with a correlation to neurobehavioral function [68]. Interestingly, tobacco smokers were found to be very resistant to ulcerative colitis [69], and the Lactobacillus reuteri recolonization was sufficient to recover metabolic function and behavioral impairment in chronically depressed mice [55, 56, 70]. Therefore, the relationship between the GM and the PAH levels in the present study seems to show that the recruitment of some GM can counteract the negative effects of the PAHs, thereby contributing to maintaining the gut ecosystem stability via a resistance phenomenon.

Another interesting finding of the present study was that PAH exposure seemed to weaken the negative association between the Cyanobacteria phylum and neurodevelopment. Cyanobacteria in the gut microbiota may produce the neurotoxins $\beta-\mathrm{N}$-methylamino$\mathrm{L}$-alanine, saxitoxin, and anatoxin- $\alpha$, which are considered to be related to the development of some neurological diseases like Alzheimer's disease [71, 72] and to aging [71]. The exact nature of the counteracting effects of the PAHs on the Cyanobacteria needs further investigation. 


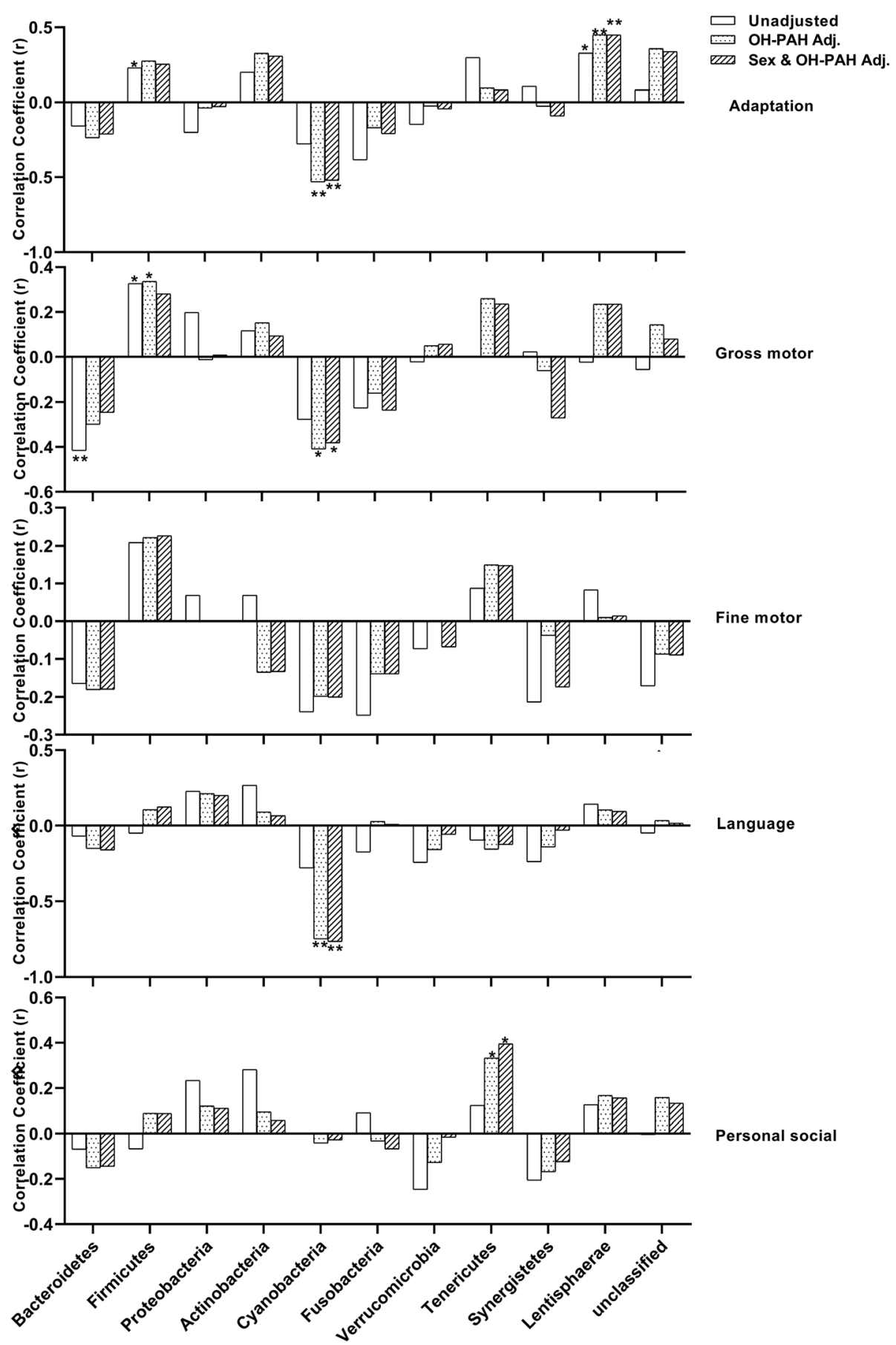

Fig. 6 The Spearman correlation coefficients between scores of five GDI behavior domains and abundance of 11 GM phyla with or without adjustment for hydroxyl PAHs and sex

In the present study, we only recruited 38 infants, of which only 25 had CBCL scores measured. Because the sample size of CBCLs was small and many partial correlation coefficients were missed, the partial correlation analysis was not performed for GM and CBCL scores. The small sample size may be our major limitation. A small sample size might cause low statistical power and a failure of detecting a significant difference. Therefore, we did not perform multivariate analysis and the correlation coefficients were presented with heatmap and hierarchical clustering. Although none of the previously reported studies calculated a sample size based on a predefined hypothesis [73], just picking the sample size might prevent us from defining an a priori probability of 
being wrong due to false negatives and false positives. Although our infants were recruited from a birth cohort, the present study was only an explorative design. The absence of evidence on the association between microbiome and the neurodevelopmental symptoms does not mean evidence of a negative association. Next, we only analyzed the composition of the GM. However, no substantial shifts in the bacterial communities of human fecal microbiota were observed in vitro [74], but acute $\mathrm{BaP}$ exposure greatly altered gut bacterial activity, regarding the volatolome (synthesis of volatile organic compounds) and the metatranscriptome, in a dosedependent manner. This implies that $\mathrm{BaP}$ can modify the gut microbial ecosystem through GM activity. Finally, GM and neurodevelopmental testing were performed at the same age, which means the nature of the cross section and unsure of the causal link between GM and neurodevelopment.

Further high-quality studies are needed to better understand this topic. The microbiome at birth, and subsequently at regular intervals until adulthood, should be characterized. The possible presence of subjects with a diagnosis of neurodevelopmental disorders should then be assessed. High-quality and comprehensive measurement methods are needed to evaluate young children's PAH exposure. Simultaneously, large differences between the dietary factors and physical activity should be included in the analyses.

\section{Conclusions}

The present study suggests that the composition of the GM is associated with neurodevelopment, and this can and should be assessed in children by 3 years of age. Simultaneously, this association was somewhat consistent with those results from the animal disease model studies or from patients with neurodevelopmental disorders. PAHs seemed to change the relative abundance of some taxa (some deleted and some recruited) to counteract the negative effects of the PAHs. To better clarify the potential role of microbiome in the association between the environmental PAH exposure and the neurodevelopmental disorders, further accurate and reliable evidence is needed.

\section{Supplementary Information}

The online version contains supplementary material available at https://doi. org/10.1186/s12887-021-02539-w.

\section{Additional file 1.}

\section{Abbreviations}

PAHs: Polycyclic aromatic hydrocarbons; GM: Gut microbiota; GDI: Gesell Development Inventory; POPs: Persistent organic pollutants; AhR: Aromatic hydrocarbon receptors; BaP: Benzo [a]pyrene; $\mathrm{CBCL}$ : Child Behavior Checklist; DQ: Developmental quotient; LOD: Limit of the detection; ADHD: Attention- deficit/hyperactivity disorder; MDD: Major depressive disorder; PCBs: Polychlorinated biphenyls; AchE: Ach level reflects acetylcholine esterase; UC: Ulcerative colitis

\section{Acknowledgements}

We would like to thank the management teams of the involved hospitals for the subjective data providing. We are also grateful to the study participants for accepting to take part in this investigation.

\section{Authors' contributions}

ZW, ZS and QZ collected the date, drafted and edited the manuscript. ZS,YS and JS collected and analyzed the date. BW, RG designed the study, and reviewed the manuscript. All the authors have read and approved the final manuscript.

\section{Funding}

This study was partly supported by grants from the National Natural Science Foundation of China (No: 81372955), Qingdao Outstanding Health Professional Development Fund (2020-2022), Qingdao Key Health Discipline Development Fund (2020-2022), the projects of medical and health technology development program in Shandong province (No: 2016WS0309), BiosTime Maternal and Infant Nutrition and Health Research Fund (2017FYH008). The funders had no role in the study design, data collection and analysis, interpretation of data, writing of the report or submission for publication.

\section{Availability of data and materials}

The datasets used and analysed during the current study are available from the corresponding author on reasonable request.

\section{Ethics approval and consent to participate}

This study was approved by the Ethics Committee and Institutional Review Board of Qingdao Centers for Disease Control and Prevention. Written informed consent was obtained from all participants's legal guardians. The authors declare that all procedures contributing to this work comply with the ethical standards of the relevant national and institutional committees on human experimentation and with the Helsinki Declaration of 1975, as revised in 2008.

\section{Consent for publication}

Not applicable.

\section{Competing interests}

The authors declare that they have no competing interests.

\section{Author details}

${ }^{1}$ Department of Nutrition and Food Hygiene, School of Public Health, Qingdao University, Qingdao, China. ${ }^{2}$ Department of Food Hygiene, Qingdao Municipality Center for Disease Control and Prevention, Qingdao Institute of Preventive Medicine, Qingdao 266033, China. ${ }^{3}$ Department of Child Health Care, Huangdao Maternity and Child Health Care Hospital of Qingdao, Qingdao 266033, China. ${ }^{4}$ Department of Environmental Health, Qingdao Municipality Center for Disease Control and Prevention, Qingdao Institute of Preventive Medicine, Qingdao 266033, China.

Received: 10 September 2020 Accepted: 4 February 2021

Published online: 17 February 2021

References

1. Rice D, Barone S Jr. Critical periods of vulnerability for the developing nervous system: evidence from humans and animal models. Environ Health Perspect. 2000;108(Suppl 3):511-33.

2. Stiles J, Jernigan TL. The basics of brain development. Neuropsychol Rev. 2010:20(4):327-48

3. Yatsunenko T, Rey FE, Manary MJ, Trehan I, Dominguez-Bello MG, Contreras $M$, et al. Human gut microbiome viewed across age and geography. Nature. 2012;486(7402):222-7.

4. Cryan J, Dinan T. Mind-altering microorganisms: the impact of the gut microbiota on brain and behaviour. Nat Rev Neurosci. 2012;13(10):701-12.

5. Sampson TR, Mazmanian SK. Control of brain development, function, and behavior by the microbiome. Cell Host Microbe. 2015;17(5):565-76. 
6. Diaz Heijtz R, Wang S, Anuar F, Qian Y, Bjorkholm B, Samuelsson A, et al. Normal gut microbiota modulates brain development and behavior. Proc Natl Acad Sci U S A. 2011;108(7):3047-52.

7. Hsiao EY, McBride SW, Hsien S, Sharon G, Hyde ER, McCue T, et al. Microbiota modulate behavioral and physiological abnormalities associated with neurodevelopmental disorders. Cell. 2013;155(7):1451-63.

8. De Palma G, Blennerhassett P, Lu J, Deng Y, Park AJ, Green W, et al. Microbiota and host determinants of behavioural phenotype in maternally separated mice. Nat Commun. 2015;6:7735.

9. Zheng P, Zeng B, Zhou C, Liu M, Fang Z, Xu X, et al. Gut microbiome remodeling induces depressive-like behaviors through a pathway mediated by the host's metabolism. Mol Psychiatry. 2016;21(6):786-96.

10. Desbonnet L, Clarke G, Shanahan F, Dinan TG, Cryan JF. Microbiota is essential for social development in the mouse. Mol Psychiatry. 2014;19(2): 146.

11. Frohlich EE, Farzi A, Mayerhofer R, Reichmann F, Jacan A, Wagner B, et al. Cognitive impairment by antibiotic-induced gut dysbiosis: analysis of gut microbiota-brain communication. Brain Behav Immun. 2016;56:140-55.

12. Savignac HM, Tramullas M, Kiely B, Dinan TG, Cryan JF. Bifidobacteria modulate cognitive processes in an anxious mouse strain. Behav Brain Res. 2015;287:59-72.

13. Kang DW, Park JG, Ilhan ZE, Wallstrom G, Labaer J, Adams JB, et al. Reduced incidence of Prevotella and other fermenters in intestinal microflora of autistic children. PLoS One. 2013;8(7):e68322.

14. Finegold SM, Dowd SE, Gontcharova V, Liu C, Henley KE, Wolcott RD, et al. Pyrosequencing study of fecal microflora of autistic and control children. Anaerobe. 2010;16(4):444-53.

15. Wang L, Christophersen CT, Sorich MJ, Gerber JP, Angley MT, Conlon MA. Increased abundance of Sutterella spp. and Ruminococcus torques in feces of children with autism spectrum disorder. Mol Autism. 2013;4(1):42.

16. Tomova A, Husarova V, Lakatosova S, Bakos J, VIkova B, Babinska K, et al. Gastrointestinal microbiota in children with autism in Slovakia. Physiol Behav. 2015;138:179-87.

17. Jiang HY, Zhou YY, Zhou GL, Li YC, Yuan J, Li XH, et al. Gut microbiota profiles in treatment-naive children with attention deficit hyperactivity disorder. Behav Brain Res. 2018:347:408-13.

18. Christian LM, Galley JD, Hade EM, Schoppe-Sullivan S, Dush CK, Bailey MT. Gut microbiome composition is associated with temperament during early childhood. Brain Behavior \& Immunity. 2015;45:118-27.

19. Stanislawski MA, Dabelea D, Wagner BD, Sontag MK, Lozupone CA, Eggesbø M. Pre-pregnancy weight, gestational weight gain, and the gut microbiota of mothers and their infants. Microbiome. 2017;5(1):113.

20. Vallès $Y$, Francino MP. Air pollution, early life microbiome, and development. Current environmental health reports. 2018;5(4):512-21.

21. Ajslev TA, Andersen CS, Gamborg M, Sørensen TI, Jess T. Childhood overweight after establishment of the gut microbiota: the role of delivery mode, pre-pregnancy weight and early administration of antibiotics. International journal of obesity (2005). 2011;35(4):522-9.

22. Collado MC, Isolauri E, Laitinen K, Salminen S. Effect of mother's weight on infant's microbiota acquisition, composition, and activity during early infancy: a prospective follow-up study initiated in early pregnancy. Am J Clin Nutr. 2010;92(5):1023-30.

23. Bhagavata Srinivasan SP, Raipuria M, Bahari H, Kaakoush NO, Morris MJ. Impacts of diet and exercise on maternal gut microbiota are transferred to offspring. Front Endocrinol. 2018;9:716.

24. Arboleya S, Suárez M, Fernández N, Mantecón L, Solís G, Gueimonde M, et al. C-section and the Neonatal Gut Microbiome Acquisition: Consequences for Future Health. Annals of Nutrition \& Metabolism. 2018;73: $17-23$.

25. Dominguez-Bello MG, Costello EK, Contreras M, Magris M, Hidalgo G, Fierer $\mathrm{N}$, et al. Delivery mode shapes the acquisition and structure of the initial microbiota across multiple body habitats in newborns. Proc Natl Acad Sci U S A. 2010;107(26):11971-5.

26. Brown LA, Khousbouei H, Goodwin JS, Irvin-Wilson CV, Ramesh A, Sheng L, et al. Down-regulation of early ionotrophic glutamate receptor subunit developmental expression as a mechanism for observed plasticity deficits following gestational exposure to benzo(a)pyrene. Neurotoxicology. 2007; 28(5):965-78.

27. Wormley DD, Ramesh A, Hood DB. Environmental contaminant-mixture effects on CNS development, plasticity, and behavior. Toxicol Appl Pharmacol. 2004;197(1):49-65.
28. Saunders CR, Das SK, Ramesh A, Shockley DC, Mukherjee S. Benzo(a)pyreneinduced acute neurotoxicity in the F-344 rat: role of oxidative stress. Journal of Applied Toxicology. 2006;26(5):427-38.

29. Perera FP, Rauh V, Whyatt RM, Tsai WY, Tang D, Diaz D, et al. Effect of prenatal exposure to airborne polycyclic aromatic hydrocarbons on neurodevelopment in the first 3 years of life among inner-city children. Environ Health Perspect. 2006;114(8):1287-92.

30. Perera FP, Tang D, Wang S, Vishnevetsky J, Zhang B, Diaz D, et al. Prenatal polycyclic aromatic hydrocarbon (PAH) exposure and child behavior at age 6-7 years. Environ Health Perspect. 2012;120(6):921-6.

31. Perera FP, Wang S, Vishnevetsky J, Zhang B, Cole KJ, Tang D, et al. Polycyclic aromatic hydrocarbons-aromatic DNA adducts in cord blood and behavior scores in New York city children. Environ Health Perspect. 2011;119(8):117681.

32. Edwards SC, Jedrychowski W, Butscher M, Camann D, Kieltyka A, Mroz E, et al. Prenatal exposure to airborne polycyclic aromatic hydrocarbons and children's intelligence at 5 years of age in a prospective cohort study in Poland. Environ Health Perspect. 2010;118(9):1326-31.

33. Yang $\mathrm{DH}$, Zhang XL, Lu XM, Zhang Q, Wang BL. Thyroid hormone disrupting activity of house dust and related pollutant. Chin J Dis Control Prev. 2017;21(1):84-8.

34. Wang BL, Pang ST, Zhang XL, Li XL, Sun YG, Lu XM, et al. Levels and neurodevelopmental effects of polycyclic aromatic hydrocarbons in settled house dust of urban dwellings on preschool-aged children in Nanjing, China. Atmospheric Pollution Research. 2014;5(2):292-302.

35. Zhang LM, Nichols RG, Correll J, Murray IA, Tanaka N, Smith PB, et al. Persistent organic pollutants modify gut microbiota-host metabolic homeostasis in mice through aryl hydrocarbon receptor activation. Environ Health Perspect. 2015;123(7):679-88.

36. Tian J, Feng Y, Fu H, Xie HQ, Jiang JX, Zhao B. The aryl hydrocarbon receptor: a key bridging molecule of external and internal chemical signals. Environ Sci Technol. 2015;49(16):9518-31.

37. Yebra-Pimentel I, Fernández-González R, Martínez-Carballo E, Simal-Gándara J. A critical review about the health risk assessment of PAHs and their metabolites in foods. Crit Rev Food Sci Nutr. 2015;55(10):1383-405.

38. Zhang $Y$, Yang $Y$, Zhang $Q$, Cui J, Rahaman A, Huang XR, et al. Effect of Benzo [a]pyrene-DNA adduct in cord blood on the neurodevelopment of 12-month-old infants in Qingdao City. Neuropsychiatr Dis Treat. 2019;15: 3351-7.

39. Xie M. The influence of polycyclic aromatic hydrocarbon exposure on the neurodevelopment of the children at 12 months age in a birth cohort. Qingdao: Qingdao University; 2017.

40. Guo Y, Senthilkumar K, Alomirah H, Moon HB, Minh TB, Mohd MA, et al. Concentrations and profiles of urinary polycyclic aromatic hydrocarbon metabolites (OH-PAHs) in several Asian countries. Environmental Science \& Technology. 2013;47(6):2932-8.

41. Achenbach TM. Manual for the child behavior checklist/2-3 and 1992 profile. Burlington: Department of Psychiatry, University of Vermont; 1992.

42. Wu L, Yao K. The revision and application of child behavior checklist for ages 2-3. Chinese Journal of Child Health Care. 1993;1 (2):81-4.

43. Song J, Zhu Y. Children's neuropsychological tests. 2nd ed. Shanghai: Shanghai Scientific and Technological Publishing Company; 1987.

44. Sram RJ, Veleminsky M Jr, Veleminsky M Sr, Stejskalová J. The impact of air pollution to central nervous system in children and adults. Neuro Endocrinology Letters. 2017;38(6):389-96.

45. Carlson AL, Xia K, Azcarate-Peril MA, Goldman BD, Ahn M, Styner MA, et al. Infant gut microbiome associated with cognitive development. Biol Psychiatry. 2018;83(2):148-59.

46. Karvonen AM, Sordillo JE, Gold DR, Bacharier LB, O'Connor GT, Zeiger RS, et al. Gut microbiota and overweight in 3-year old children. Int J Obes. 2019;43(4):713-23.

47. Eckburg PB, Bik EM, Bernstein CN, Purdom E, Dethlefsen L, Sargent M, et al. Diversity of the human intestinal microbial flora. Science. 2005;308(5728):1635-8.

48. Schroeder BO, Backhed F. Signals from the gut microbiota to distant organs in physiology and disease. Nat Med. 2016;22(10):1079-89.

49. Dinan TG, Cryan JF. Microbes, immunity, and behavior: psychoneuroimmunology meets the microbiome. Neuropsychopharmacology. 2017;42(1):178.

50. Erny D, de Angelis ALH, Jaitin D, Wieghofer P, Staszewski O, David E, et al. Host microbiota constantly control maturation and function of microglia in the CNS. Nat Neurosci. 2015;18(7):965 
51. Abdel-Haq R, Schlachetzki JCM, Glass CK, Mazmanian SK. Microbiomemicroglia connections via the gut-brain axis. J Exp Med. 2019;216(1):41-59.

52. Bajaj JS, Ridlon JM, Hylemon PB, Thacker LR, Heuman DM, Smith S, et al. Linkage of gut microbiome with cognition in hepatic encephalopathy. Am J Physiol Gastrointest Liver Physiol. 2012;302(1):G168-75.

53. Bruce-Keller AJ, Salbaum JM, Luo M, Blanchard E IV, Taylor CM, Welsh DA, et al. Obese-type gut microbiota induce neurobehavioral changes in the absence of obesity. Biol Psychiatry. 2015;77(7):607-15.

54. Magnusson KR, Hauck L, Jeffrey BM, Elias V, Humphrey A, Nath R, et al. Relationships between diet-related changes in the gut microbiome and cognitive flexibility. Neuroscience. 2015;300:128-40.

55. Wang S, Blazer DG. Depression and cognition in the elderly. Annu Rev Clin Psychol. 2015;11:331-60.

56. Bravo JA, Forsythe P, Chew MV, Escaravage E, Savignac HM, Dinan TG, et al. Ingestion of Lactobacillus strain regulates emotional behavior and central GABA receptor expression in a mouse via the vagus nerve. Proc Natl Acad Sci U S A. 2011;108(38):16050-5.

57. Kelly JR, Borre Y, O'Brien C, Patterson E, El Aidy S, Deane J, et al. Transferring the blues: Depression-associated gut microbiota induces neurobehavioural changes in the rat. J Psychiatr Res. 2016;82:109-18.

58. Wang LJ, Yang CY, Chou WJ, Lee MJ, Chou MC, Kuo HC, et al. Gut microbiota and dietary patterns in children with attention-deficit/ hyperactivity disorder. Eur Child Adolesc Psychiatry. 2019;29(3):287-97.

59. Xu M, Xu X, Li J, Li F. Association between gut microbiota and autism Spectrum disorder: a systematic review and meta-analysis. Frontiers in psychiatry. 2019;10:473

60. Rota M, Bosetti C, Boccia S, Boffetta P, La Vecchia C. Occupational exposures to polycyclic aromatic hydrocarbons and respiratory and urinary tract cancers: an updated systematic review and a meta-analysis to 2014. Arch Toxicol. 2014;88(8):1479-90

61. Van de Wiele T, Vanhaecke L, Boeckaert C, Peru K, Headley J, Verstraete W, et al. Human colon microbiota transform polycyclic aromatic hydrocarbons to estrogenic metabolites. Environ Health Perspect. 2005;113(1):6-10.

62. Ribiere C, Peyret P, Parisot N, Darcha C, Dechelotte PJ, Barnich N, et al. Oral exposure to environmental pollutant benzo a pyrene impacts the intestinal epithelium and induces gut microbial shifts in murine model. Sci Rep. 2016; 6:11.

63. Caspani G, Kennedy S, Foster JA, Swann J. Gut microbial metabolites in depression: understanding the biochemical mechanisms. Microb Cell. 2019; 6(10):454-81.

64. Lefever DE, Xu J, Chen YJ, Huang GM, Tamas N, Guo TL. TCDD modulation of gut microbiome correlated with liver and immune toxicity in streptozotocin (STZ)-induced hyperglycemic mice. Toxicol Appl Pharmacol. 2016;304:48-58

65. Chi YL, Lin Y, Zhu HM, Huang QS, Ye GZ, Dong SJ. PCBs-high-fat diet interactions as mediators of gut microbiota dysbiosis and abdominal fat accumulation in female mice. Environ Pollut. 2018:239:332-41.

66. Stanaszek P, Snell J, O'Neill J. Isolation, extraction, and measurement of acetylcholine from Lactobacillus plantarum. Appl Environ Microbiol. 1977; 34(2):237-9

67. Cunha I, García L, Guilhermino L. Sea-urchin (Paracentrotus lividus) glutathione S-transferases and cholinesterase activities as biomarkers of environmental contamination. Journal of Environmental Monitoring. 2005; 7(4):288-94

68. Qiao N, Zhang H, Li X, Li M. Benzo [a]pyrene-induced neurobehavioral function and neurotransmitter alterations in coke oven workers. Occupational \& Environmental Medicine. 2010;67(7):444-8.

69. Lunney P, Leong R. Review article: ulcerative colitis, smoking and nicotine therapy. Aliment Pharmacol Ther. 2012;36:997-1008.

70. Marin I, Goertz J, Ren T, Rich S, Onengut-Gumuscu S, Farber E, et al. Microbiota alteration is associated with the development of stress-induced despair behavior. Sci Rep. 2017;7:43859.

71. Brenner SR. Blue-green algae or cyanobacteria in the intestinal micro-flora may produce neurotoxins such as Beta-N-Methylamino-L-Alanine (BMAA) which may be related to development of amyotrophic lateral sclerosis, Alzheimer's disease and Parkinson-dementia-complex in humans and equine motor neuron disease in horses. Med Hypotheses. 2013;80(1):103.

72. Banack SA, Caller TA, Stommel EW. The cyanobacteria derived toxin Beta-Nmethylamino-L-alanine and amyotrophic lateral sclerosis. Toxins (Basel). 2010;2(12):2837-50
73. Lacorte E, Gervasi G, Bacigalupo I, Vanacore N, Raucci U, Parisi P. A systematic review of the microbiome in children with neurodevelopmental disorders. Front Neurol. 2019;10:727.

74. Defois C, Ratel J, Denis S, Batut B, Beugnot R, Peyretaillade E, et al. Environmental pollutant Benzo a Pyrene impacts the volatile Metabolome and Transcriptome of the human gut microbiota. Front Microbiol. 2017;8:12.

\section{Publisher's Note}

Springer Nature remains neutral with regard to jurisdictional claims in published maps and institutional affiliations.
Ready to submit your research? Choose BMC and benefit from:

- fast, convenient online submission

- thorough peer review by experienced researchers in your field

- rapid publication on acceptance

- support for research data, including large and complex data types

- gold Open Access which fosters wider collaboration and increased citations

- maximum visibility for your research: over $100 \mathrm{M}$ website views per year

At BMC, research is always in progress.

Learn more biomedcentral.com/submissions 